\title{
Inflammatory response of two invasive techniques in the mouse with collagenase induced tendinopathy
}

\author{
García Vidal J.A. ${ }^{1}$ Pelegrín Vivancos P. ${ }^{2} \quad$ Escolar Reina P. ${ }^{1}$ Medina i Mirapeix F. ${ }^{1}$ \\ ${ }^{1}$ Department of Physiotherapy. Universidad de Murcia, Murcia, Spain \\ 2 Instituto Murciano de Investigacion Biosanitaria (IMIB), \\ Murcia, Spain \\ Rev Fisioter Invasiva 2019;2:80.
}

\begin{abstract}
Background Percutaneous needle electrolysis (PNE) is a physiotherapy technique which has demonstrated its effectiveness for the treatment of tendinopathies and muscle pathologies. All the authors consulted base its therapeutic effect on the capacity to produce an important inflammatory response during the initial phase of the regenerative process. However, presently, the histological effects of the technique on tendinopathies are unknown. The aim of this study was to histologically examine the inflammatory response provoked by the PNE versus dry needling (DN) in mice with collagenase-induced tendinopathy.

Material and Methods In order to achieve the proposed aim, a murine model was used with a total of $18 \mathrm{C} 57 \mathrm{BL} / 6 \mathrm{~J}$ mice. One week prior to the intervention, collagenaseinduced tendinopathy was performed in the common calcaneal tendons of all mice. Once the pathology was established, they received DN in the left common calcaneus tendon, whereas, on the right, they received treatment with PNE. Groups of animals were sacrificed at 3,7 and 14 days to evaluate the histological evolution of injuries. Samples of the common calcaneal tendon were taken and were later fixed in formalin, processed, and included in paraffin blocks. Sections of 3 microns thick were performed, which were dyed using hematoxylin-eosin for conventional histopathological examination.

Results In both groups, tissue damage was observed with collagen fragmentation and the presence of fibroblasts. Dry needling of the common calcaneal tendon induced the presence of an acute inflammatory infiltrate (characterized by the presence of polymorphonuclear neutrophils and macrophages) at the level of the peritenon and

\section{Keywords}

- collagenase

- tendinopathy

- mouth

- electrolysis

- inflammation adjacent fat tissue which was detectable after day 3 , and notable after days 7 and 14 . The application of PNE induced a similar effect, although on day 14 , the inflammatory infiltrate of PNE was more evident, compared to DN.

Conclusion The proposed in vivo murine model has demonstrated to be useful for the study of the evolution of the first phases of the regenerative process induced by both techniques. The histopathological results reveal that PNE generates a pro-inflammatory stimulus which is superior to DN on day 14 .
\end{abstract}

DOI https://doi.org/ $10.1055 / \mathrm{s}-0039-3401862$. ISSN 2386-4591.
Copyright @ 2019 by Thieme Revinter Publicações Ltda, Rio de Janeiro, Brazil
License terms

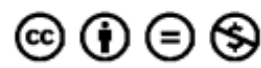

